\title{
An Interval Type-2 Fuzzy Cellular Automaton Model for Traffic Flow
}

\author{
Jie Dong, Xiaomei Zhang \\ School of Control Science and Engineering, Dalian University of Technology, Dalian, 116024, China \\ E-mail: dongjie@dlut.edu.cn
}

\begin{abstract}
On the basis of Nagel-Schreckenberg model, this paper presents a modified fuzzy cellular automaton model for one-lane highway traffic. An interval type-2 fuzzy logic system is designed to evaluate the randomization parameter of a vehicle based on the spatial headway and speed difference. Some basic qualitative results and the fundamental diagram of our new model are got through computer numerical simulations. The experimental results show that our new model is able to reproduce some of the real macroscopic traffic characteristics such as stop-and-go waves, hysteresis and metastability phenomena.
\end{abstract}

Keywords-traffic flow; cellular automaton model; interval type-2 fuzzy sets; randomization deceleration probability

\section{INTRODUCTION}

Traffic flow model has been studied to improve the control, management and organization of traffic system since a long time ago. After the Kinematics Model [1,2], Car-following Model [3,4] and Gas-Kinetics Theory [5,6], cellular automaton (CA) model [7] has been widely applied in study of traffic flow in recent years. CA model is a useful tool for simulation of nonlinear complex systems. NaSch model is the most popular cellular automaton model for traffic flow.

In 1992, Nagel and Schreckenberg proposed the NaSch model [8] based on the Rule 184 CA [9]. The model is simple, but is able to describe some real traffic phenomena. Therefore, NaSch model is regarded as a minimum model to reproduce the basic features of traffic flow. However, real traffic flow has hysteresis, metastability and other complex phenomena and characteristics, which NaSch model can not reproduce. In order to reproduce these phenomena, various modified models have been proposed. M. Takayasu et al. [10] and S. C. Benjamin et al. [11] introduced slow-to-start rules into NaSch model. R. Barlovic et al. proposed a simple extension NaSch model by introducing a velocitydependent randomization (VDR) [12]. X. B. Li et al. presented a velocity effect (VE) model [13], in which model the positions and velocities of the two cars are considered as critical factors. These above models are all able to reproduce hysteresis and metastability phenomena.

Traffic flow shows some randomness $[14,15,16]$, and has a certain ambiguity. In a simple NaSch model, applying the same randomization deceleration probability to all the vehicles is unreasonable. To make the traffic flow model based on cellular automaton more close to reality, an interval type-2 fuzzy logic system (IT2 FLS) is designed and integrated into the traffic flow model in this paper. This system handles the uncertainties of the influencing factors' randomness with more parameters and more degrees of freedom than with a type-1 fuzzy logic system (T1 FLS). The new model is called headway-velocity-dependentrandomization (HVDR) model. Spatial headway and velocity difference, two main driving factors, are the inputs of IT2 FLS. The output is the randomization deceleration probability of the current vehicle at the next time step. The computer simulation results show that the new model is able to reproduce some of the real macroscopic traffic characteristics such as stop-and-go waves, hysteresis and metastability phenomena.

\section{NASCH MODEL}

In the classical NaSch model, $x_{n}(t)$ and $x_{n-1}(t)$ denote the location of the vehicle $n$ and the location of the vehicle $n-1$ at the time step $t$ respectively, vehicle $n-1$ runs just in front of the vehicle $n . d_{n}(t)$ denotes the spatial headway of the vehicle $n$ at the time step $t, d_{n}(t)=x_{n-1}(t)-x_{n}(t)-\mathbf{1}$. Car $n$ moves with the velocity of $v_{n}(t)$ between zero and $v_{\max }$. What's more, $p_{n}(t)$ denotes the randomization parameter of vehicle $n$ at time step $t$ and $\Delta v_{n}(t)$ stands for the result that $v_{n-1}(t)$ subtracted from $v_{n}(t)$. The parallel update rules of NaSch model are as follows:

- Acceleration.

If $v_{n}(t)<v_{\max }$, the speed of vehicle $n$ will increase by one. And $v_{n}(t)$ remains unchanged if the car has reached the maximum velocity already. i.e. $v_{n}(t+1) \rightarrow \min \left(v_{n}(t)+1, v_{\max }\right)$.

- Deceleration. If $v_{n}(t+\mathbf{1}) \geq d_{n}(t)$, the speed of vehicle $n$ will decrease to $d_{n}(t)$. Otherwise, $v_{n}(t+1)$ remains unchanged. i.e. $v_{n}(t+\mathbf{1}) \rightarrow \min \left(v_{n}(t+\mathbf{1}), d_{n}(t)\right)$.

- Randomization.

If $v_{n}(t)>\mathbf{0}$, the speed of vehicle $n$ will decrease with the probability $p$. But the speed won't change if $v_{n}(t)=\mathbf{0}$. i.e. $v_{n}(t+\mathbf{1}) \rightarrow \max \left(v_{n}(t+\mathbf{1})-\mathbf{1 , 0}\right)$.

- Movement

Every vehicle will move with its new velocity determined by the foregoing three steps. i.e. $x_{n}(t+1) \rightarrow x_{n}(t)+v_{n}(t+1)$.

NaSch model is a minimal model which is able to qualitatively reproduce some of the traffic features such as congestion, flow-density relation and the stop-and-go waves. 


\section{INTERVAL TYPE-2 FUZZY LOGIC SYSTEM}

In the classical NaSch model, the randomization parameter of a vehicle is a constant between zero and one. That's not consistent with the actual traffic situation. We can use fuzzy sets to depict the fuzziness of traffic flow, and make the simulation results more close to actual traffic. Type-1 fuzzy sets [17] can be used in this circumstance, but the membership functions of type-1 fuzzy sets are certain, and commonly rooted in the knowledge of experts and skilled operators. It's greatly subjective and indeterminate. Type-2 fuzzy sets [18] are also able to model such uncertainties. Unfortunately, type-2 fuzzy sets are more difficult to use and understand than type-1 fuzzy sets. Interval type-2 fuzzy sets [19] are able to depict fuzziness and uncertainty with lower calculation complexity.

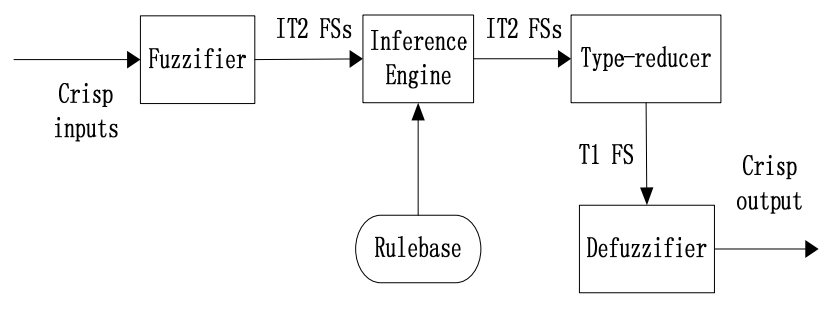

Figure 1. An Interval Type-2 Fuzzy Logic System.

Fig. 1 shows the schematic diagram of the IT2 FLS [20]. It is similar to its T1 counterpart, the major difference is that at least one of the FSs in the rule base is an IT2 FS. Hence, the outputs of the inference engine are IT2 FSs, and a typereducer is needed to convert them into a T1 FS before defuzzification can be carried out. In HVDR model, the randomization parameter $p$ changes at every time step. The acceleration, deceleration and movement rules remain unchanged just like the NaSch model. The spatial headway and velocity difference are considered as two critical factors that affect the behaviors of drivers. They are the inputs of the interval type-2 fuzzy logic system, and the output is the randomization probability at the coming time step.

\section{A. Membership functions for the input and output variables}

The experimental simulation conditions are determined according to the actual traffic situations. The value of spatial headway $d(t)$ can be enlarged to be in $[0,50]$ reasonably. Car $n$ moves on the lattice with the velocity of $v_{n}(t)$ between zero and $v_{\max }$, where $v_{\max }=5$.

In this paper, the fuzzy sets of input and output variables are interval type- 2 fuzzy sets. MFs for $d(t)$ and $\Delta v(t)$ are determined according to the MFs in paper [21] and enough simulation results. $d(t)$ is characterized by eleven interval type-2 fuzzy sets (fig.2), and $\Delta v(t)$ is characterized by six interval type-2 fuzzy sets (fig.3). The centroids of consequents can be seen in TABLE I. The centroids are all located in the interval $[0,1]$. The neighboring ones overlap partially to make the randomization parameters more close under similar conditions.

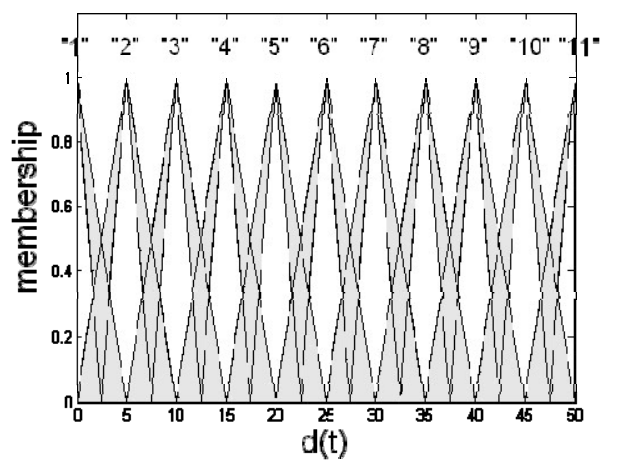

Figure 2. MFs for $d(t)$.

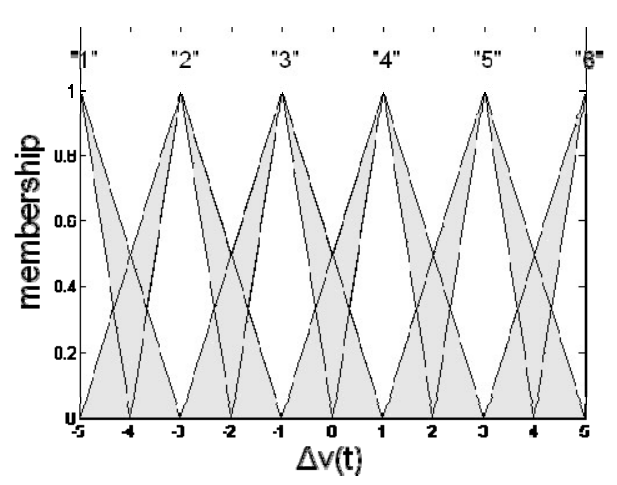

Figure 3. MFs for $\Delta v(t)$.

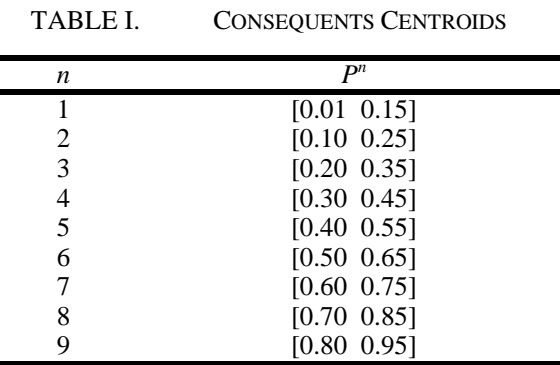

\section{B. Fuzzy rulebase}

The IT2 FLS is characterized by IF-THEN rules. The rulebase is designed according to the assumptions as follows:

1) There is an effective distance. If a vehicle is out of the effective distance, the effection on the vehicle by its preceding vehicle can be ignored. In this situation, $p$ is defined as 0.25 to depict the effection of some other factors.

2) Within the effective distance, randomization deceleration probability increases with the increase of velocity difference and decreases with the decrease of velocity difference. Randomization deceleration probability increases with the decrease of spatial headway and decreases with the increase of spatial headway.

3) Within the effective distance, both the spatial headway and the velocity difference should be considered to get a relatively reasonable randomization parameter. 
TABLE II shows the rulebase of the IT2 FLS based the foregoing conditions. The first line is linguistic variable $\Delta v(t)$, the first column is linguistic variable $d(t)$, and the intersection is linguistic variable $p(t+\mathbf{1})$ determined by spatial headway and velocity difference. The IF-THEN rule form is that: IF $d(t)$ is 3 and $\Delta v(t)$ is 6, THEN $p(t+\mathbf{1})$ is 8 .

TABLE II. FUZZY RULEBASE

\begin{tabular}{c|cccccc}
\hline$\Delta v(t)$ & & & & & & \\
$d(t)$ & & 2 & 3 & 4 & 5 & 6 \\
\hline 1 & & & & & & \\
2 & 6 & 7 & 8 & 8 & 9 & 9 \\
3 & 6 & 6 & 7 & 7 & 8 & 9 \\
4 & 5 & 5 & 6 & 6 & 7 & 7 \\
5 & 4 & 5 & 5 & 6 & 6 & 7 \\
6 & 4 & 4 & 5 & 5 & 6 & 6 \\
7 & 3 & 4 & 4 & 5 & 5 & 6 \\
8 & 3 & 3 & 4 & 4 & 5 & 5 \\
9 & 2 & 3 & 3 & 4 & 4 & 5 \\
10 & 2 & 2 & 3 & 3 & 4 & 4 \\
11 & 1 & 2 & 2 & 3 & 3 & 4 \\
\hline
\end{tabular}

IV. Simulation RESUltS

Single lane cellular automaton models are generally defined on a one-dimensional lattice of $L$ sites with periodic boundary conditions. In the computer simulations of HVDR model, the real length of the single lane is $15 \mathrm{~km}$. There are 2000 cells, each cell is $7.5 \mathrm{~m}$ in reality. Each cell is either covered with a vehicle or totally unoccupied. The density $\rho$ is defined as $N / L$, where $N$ denotes the number of vehicles. From right to left, the $N$ cars are numbered orderly from 1 to $N$. One time step is $1 \mathrm{~s}$ and velocity unit is roughly $27 \mathrm{~km} / \mathrm{h}$. It is assumed that $v_{\max }$ equals 5, which implies a maximum velocity of $135 \mathrm{~km} / \mathrm{h}$. In each time step, the velocity of every vehicle is renewed according to update rules and the randomization parameter of each car is also calculated according the fuzzy rules. Relevant data are collected after 10000 time steps' simultaneous evolution with periodic boundary conditions.

Fig.4 shows the space-time diagrams of HVDR model. The horizontal direction is space from left to right and the vertical direction is time from above to below. The white spot shows that the cell is occupied by a vehicle, and the black spot shows that the cell is unoccupied. Fig.4(a) depicts the space-time diagram of free flow at a low density, $\rho=0.05$. Fig.4(b) depicts the space-time diagram of stopand-go waves at a relatively higher density, $\rho=0.4$. It can be seen that HVDR model is able to reproduce the free flow and stop-and-go waves.

What's more, under a specific condition, HVDR model can reproduce an important characteristic of traffic flow, "hysteresis", which cannot be reproduced in the NaSch model at all. Fig.5 depicts the fundamental diagrams of NaSch model and HVDR model. There are two branches in the fundamental diagram of HVDR model during the density range [0.06, 0.24]. The upper branch is calculated by adding cars to a homogeneous state, while the lower branch is calculated by removing cars from a jammed state. Metastability exists during the density range [0.06, 0.24]: the traffic flow is free flow or crowded flow.

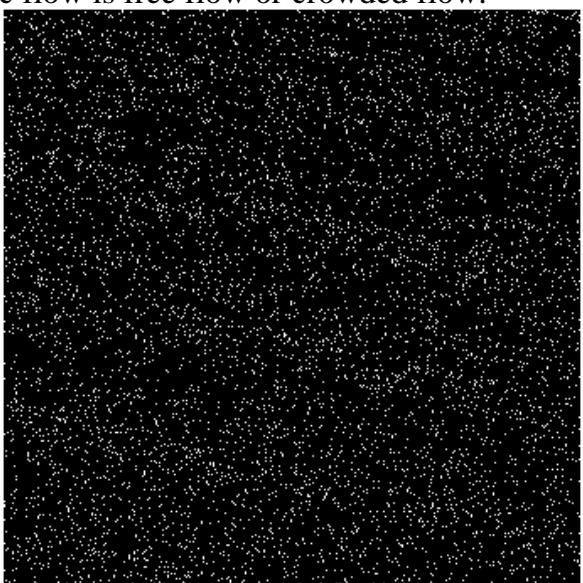

(a) $v_{\max }=5, \rho=0.05$

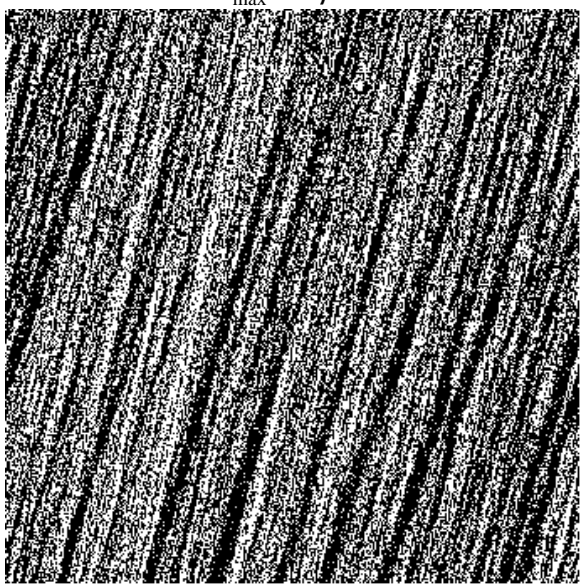

(b) $v_{\max }=5, \rho=0.4$

Figure 4. Space-time diagrams of HVDR model.

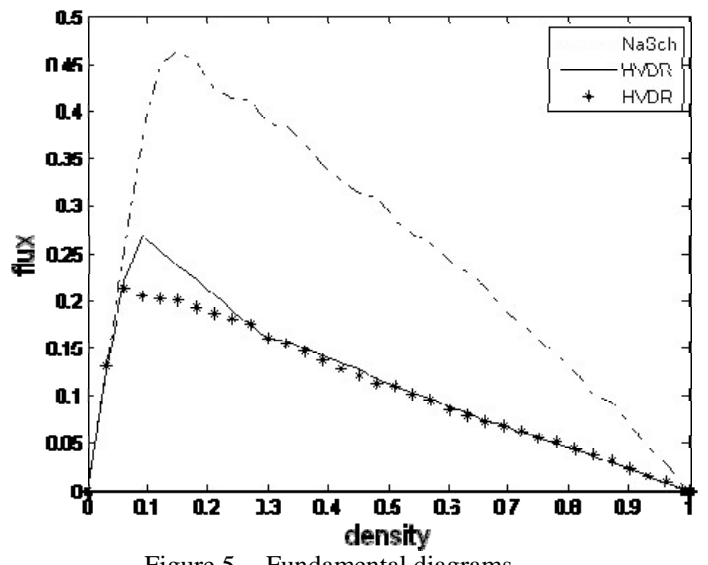

Figure 5. Fundamental diagrams.

Through analyzing the time series of randomization parameter in HVDR model, we can find the change rules of randomization parameter at different densities. The upper part of fig.6(a) is the randomization parameter time series of a vehicle at a density of 0.05 in 1000 time steps. The traffic is free flow. The spatial headway and velocity difference are 
both relatively large, so the calculated randomization parameter is small and in [0.35, 0.05]. The lower part of fig.6(a) is the randomization parameter time series of a vehicle at a density of 0.8 . The traffic is crowded. The spatial headway and velocity difference are both relatively small, so the calculated randomization parameter is large and in [0.80, 0.90]. Fig.6(b) depicts the time series of randomization parameter at a density of 0.4 . The traffic is in stop-and-go waves. Spatial headway and velocity difference change constantly, so does the calculated randomization parameter. It's basically consistent with the actual situation.

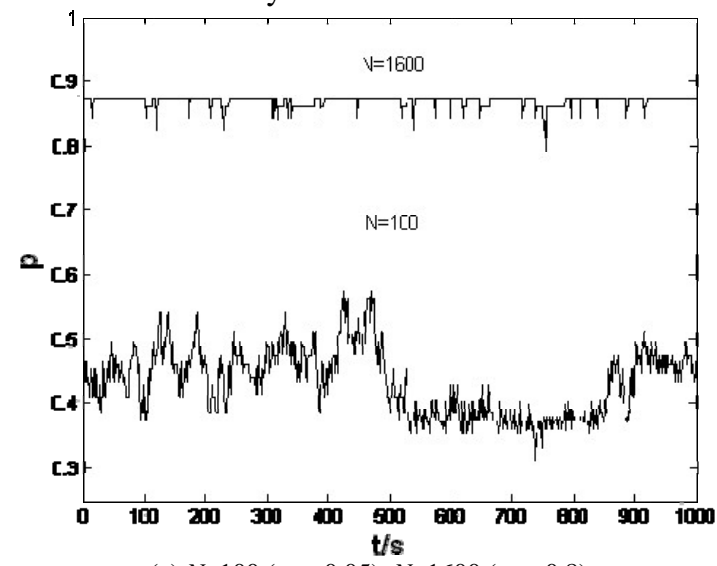

(a) $N=100(\rho=0.05), N=1600(\rho=0.8)$

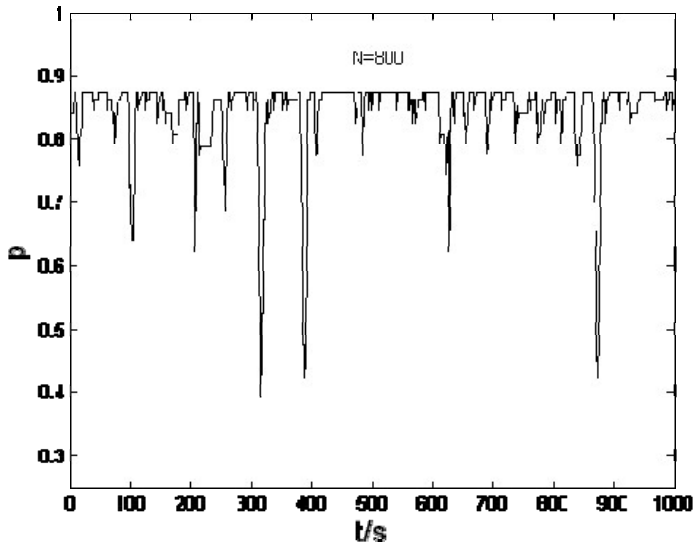

(b) $N=800(\rho=0.4)$

Figure 6. Time series of randomization parameter in HVDR model.

\section{CONCLUSIONS}

In this paper we present HVDR model for one-lane traffic flow based on interval type-2 fuzzy sets. In the model, randomization parameter of a vehicle is determined more reasonably by spatial headway and velocity difference instead of a constant between zero and one. The calculated randomization parameters are basically consistent with the actual traffic situation. The experimental results show that our new model is able to reproduce some of the real macroscopic traffic characteristics such as stop-and-go waves, hysteresis and metastability phenomena. The work done in this paper is just a try to bring the interval type-2 fuzzy viewpoint into the traffic flow research, more creative and further researches are expected in the future.

\section{ACKNOWLEDGMENT}

This work was financially supported by the National Natural Science Foundation of China with Grant No. 61004040.

\section{REFERENCES}

[1] M. J. Lighthill, G. B. Whitham, "On kinematic waves: a theory of traffic flow on long crowed roads,” Proc. Roy. Soc. London, vol. 229, pp. 317-345, 1955.

[2] C. F. Daganzo, "Requiem for second-order fluid approximations of traffic flow,” Transpn. Res. B, vol. 29, pp. 277-286, 1995.

[3] L. A. Pipes, "An operational analysis of traffic dynamics,” J. Appl. Phys., vol. 24, pp. 274-281, 1953.

[4] M. Treiber, A. Hennecke, D. Helbing, "Congested traffic states in empirical observations and microscopic simulations," Phys. Rev. E, vol. 62, pp. 1805-1824, 2000

[5] I. Prigogine, R. Herman, "Kinetic theory of vehicular traffic," American Elsevier, New York, pp. 17-54, 1971.

[6] S. L. Paveri-Fontana, “On Boltzmann-like treatments for traffic flow: a critical review of the basic model and an alternative proposal for dilute traffic analysis,” Transpn. Res. B, vol. 9, pp. 225-235, 1975.

[7] B. Jia, Z. Y. Gao, K. P. Li, Models and Simulations of Traffic System Based on the Theory of Cellular Automaton. Science Press, Beijing, 2007.

[8] K. Nagel, M. Schreckenberg, “A cellular automaton model for freeway traffic,” J. Phys. (I), vol. 2, pp. 2221-2229, 1992.

[9] S. Wolfram, “Theory and Applications of Cellular Automata,” World Scientific, Singapore, pp. 485-557, 1986.

[10] M. Takayasu, H. Takayasu, “ $1 / \mathrm{f}$ noise in a traffic model,” Fractals, vol. 1, pp. 860-866, 1993.

[11] S. C. Benjamin, N. F. Johnson, P. M. Hui, "Cellular automata models of traffic flow along a highway containing a junction,” J. Phys. A: Math. \& Gen., vol. 29, pp. 3119-3127, 1996.

[12] R. Barlovic, L. Santen, A. Schadschneider, "Metastable states in cellular automata for traffic flow,” Eur. Phys. J. B, vol. 5, pp. 793-800, 1998.

[13] X. B. Li, Q. S. Wu, R. Jiang, "Cellular automaton model considering the velocity effect of a car on the successive car,” Phys. Rev. E, vol. 64, pp. 066128(1-4), 2001.

[14] X. J. Kong, Z. Y. Gao, K. P. Li, “A two-lane cellular automata model with influence of next-nearest neighbor vehicle,” Commun. Theor. Phys., vol. 45, pp. 657-662, 2006.

[15] L. J. Peng, R. Kang, "One-dimensional cellular automaton model of traffic flow considering drivers’ features,” Acta Phys. Sin., vol. 58, pp. 830- 835, 2009.

[16] J. X. Ding, H. J. Huang, T. Q. Tang, “A cellular automaton model of traffic considering the dynamic evolution of velocity randomization probability,” Acta Phys. Sin., vol. 58, pp. 7591-7595, 2009.

[17] Z. H. Lv, H. Jin, P. P. Yuan, "The theory of triangle type-2 fuzzy sets," Ninth IEEE International Conference on Computer and Information Technology(CIT 09), IEEE Press, Oct. 2009, pp. 57-62.

[18] L. A. Zadeh, "The concept of a linguistic variable and its application to approximate reasoning-1,” Inf. Sci., vol. 8, pp. 199-249, 1975.

[19] L. R. Cecilia, C. Oscar, R. D. Antonio, "Interval type-2 fuzzy logic system to simulate the environment resources stochasticity affecting the growth of a population," IEEE International Conference on Fuzzy Systems(FUZZ-IEEE 09), IEEE Press, Aug. 2009, pp. 726-731.

[20] Q. L. Liang, J. M. Mendel, "Interval type-2 fuzzy logic systems: theory and design,” IEEE Trans. on Fuzzy Syst., vol. 8, pp. 535-550, 2000.

[21] Y. G. Gong, L. Liu, “A Fuzzy Cellular Automaton Model Based On NaSch Model,” 2010 2nd International Conference on Signal Processing Systems (ICSPS), July 2010, pp. V2-518-V2-522. 